\title{
Gazeover - Exploring the UX of Gaze-triggered Affordance Communication for GUI Elements
}

\author{
Ilhan Aslan, Michael Dietz, and Elisabeth André \\ Human-Centered Multimedia Lab, Augsburg University \\ Augsburg, Germany \\ lastname@hcm-lab.de
}

\begin{abstract}
The user experience (UX) of graphical user interfaces (GUIs) often depends on how clearly visual designs communicate/signify "affordances", such as if an element on the screen can be pushed, dragged, or rotated. Especially for novice users figuring out the complexity of a new interface can be cumbersome. In the "past" era of mouse-based interaction mouseover effects were successfully utilized to trigger a variety of assistance, and help users in exploring interface elements without causing unintended interactions and associated negative experiences. Today's GUIs are increasingly designed for touch and lack a method similiar to mouseover to help (novice) users to get acquainted with interface elements. In order to address this issue, we have studied gazeover, as a technique for triggering "help or guidance" when a user's gaze is over an interactive element, which we believe is suitable for today's touch interfaces. We report on a user study comparing pragmatic and hedonic qualities of gazeover and mouseover, which showed significantly higher ratings in hedonic quality for the gazeover technique. We conclude by discussing limitations and implications of our findings.
\end{abstract}

\section{CCS CONCEPTS}

\section{- Human-centered computing $\rightarrow$ Interaction techniques;}

\section{KEYWORDS}

Gaze, user experience

\section{ACM Reference Format:}

Ilhan Aslan, Michael Dietz, and Elisabeth André. 2018. Gazeover - Exploring the UX of Gaze-triggered Affordance Communication for GUI Elements. In 2018 International Conference on Multimodal Interaction (ICMI '18), October 16-20, 2018, Boulder, CO, USA. ACM, New York, NY, USA, 5 pages. https: //doi.org/10.1145/3242969.3242987

\section{INTRODUCTION}

Screen-based graphical interfaces still dominate the interface landscape in human-computer interaction (HCI) and in contrast to the beginnings when interfaces were designed to be used with a mouse device, today's graphical interfaces are often touch-sensitive and

This is the author's version of the work. It is posted here for your personal use. Not for redistribution. The definitive Version of Record was published in:

ICMI '18, October 16-20, 2018, Boulder, CO, USA

(c) 2018 Association for Computing Machinery. ACM ISBN 978-1-4503-5692-3

https://doi.org/10.1145/3242969.3242987 implement the direct manipulation concept introduced by Schneiderman [35] in a stricter manner. The benefits of direct manipulation interfaces are undeniable and fellow researcher, such as Hutchins and colleagues [19] have explored in detail the technique's advantages and disadvantages.

Of great relevance for direct manipulation interfaces is the idea of visual affordances, which is associated with directly perceiving (inter)action opportunities. Interestingly, the idea of affordances has been one of the most pragmatic but also controversial topics in HCI (e.g., [4, 14, 34]), since with graphical interfaces it can be differentiated between perceivable and actual action opportunities (e.g., a virtual button with a 3D effect only "pretends" that it can be physically pushed). Thus, one of the main challenges for designers of direct manipulation interfaces is associated with having to represent/signify action potentials on a flat $2 \mathrm{D}$ screen, which most people have learned (possibly growing in different cultures) through everyday practices in the real world and are often based on manipulating physical 3D objects and controllers.

In addition to the challenge of designing good "affordance signifiers" for direct manipulation interfaces, and consequently designing interfaces, which are easy to learn and use (i.e., interfaces with good pragmatic qualities), designers also have to consider the aesthetics of interfaces and interactions (i.e., hedonic qualities, such as if the interface is perceived as beautiful and if the interaction is desirable). Both quality dimensions, pragmatic and hedonic, constitute to the overall UX of a user interface [18]; however, the dimensions are somewhat independent and may be in contrast to each other. Consequently, a direct manipulation interface which is beautiful might not "function" well, because users (especially novice users) have difficulties to perceive action opportunities (e.g., on flat graphical designs it is often difficult to depict interactive elements). For mouse-based interfaces, tooltips that are triggered by mouseover events have been popular and provided designers more freedom while allowing users to explore the functionality of a graphical interface in a safe manner. A similar method is missing for today's touch interfaces because most touch interfaces do not provide a pre-touch event, but solutions may be provided by multi-modal techniques, such as a proposition by Aslan and André [3] to use pre-touch hand/finger proxemics (e.g., the 3D distance of fingers above a screen to touch targets) to trigger help with communicating a touch targets "affordances".

In this paper, we study an alternative technique based on eye gaze, arguing that the task of visually exploring functionalities of a graphical interface maps well (and more natural) to eye gaze as an interaction modality. Before we report on a user study with 12 participants, which demonstrates that the UX of the gazeover 
technique is even superior to the mouseover technique, we provide in the following section background in gaze-based interaction.

\section{BACKGROUND IN GAZE-INTERACTION}

\subsection{Gaze as an analytical measure}

Human vision is a dynamic process during which a person's gaze is directed towards certain points or regions of interest [24]. Thereby the current cognitive processes are reflected in specific eye movement patterns, which can give insights into the thoughts and intentions of the person [23]. This correlation was first investigated by Buswell [7] in 1935, where he showed the influence of viewing tasks on eye movement responses by giving participants different viewing instructions when looking at a picture. Since then, a lot of research has focused on the analysis of eye movements in various activities and applications, such as the usability tests of web pages [9], menu-based interfaces [1], and graphical layouts [8] Others have analyzed gaze to detect user activities and tasks (e.g., $[6,11,12])$, or emotional state (e.g., $[2,41])$.

\subsection{Gaze as an input modality}

Eye movements can also be used as an input modality. For instance, a very simple approach is to let the users control a cursor with their eyes. This can be useful for disabled people who cannot use a mouse or keyboard with their hands [20] or for visually impaired users who want to control the direction of a sonification system [10]. As shown in [39], using gaze as input method can also be beneficial for users without impairments due to the faster pointing times compared to traditional mouse movements. However, using the eyes to select and control graphical interfaces can lead to the socalled "Midas touch" problem [22], where viewed items are selected unintentionally. The reason for this is that the same modality is used to perceive and control the content, which cannot be distinguished by the system [29].

One solution to this problem is to introduce a short delay called "dwell-time", before interpreting the gaze-behavior as intended interaction [16, 27]. Although long fixations are usually perceived as straining and uncomfortable, the usage of short and adjustable dwell-times has proven to be effective [28, 33].

\subsection{Gaze in multimodal interaction}

Gaze input can easily be combined with other modalities, for example to confirm selections. This can be achieved with various triggers such as hand gestures [40], keyboard strokes [25], touch inputs [37], voice commands [32] or even muscle activities [30]. An exemplary domain where gaze is integrated with other modalities is video games in which gaze input is mostly used to target enemies [36] or objects [15], while the final selection is confirmed with a mouse click. Others (e.g., [31]) have used gaze to integrate onlookers into the game play. Further implicit interaction approaches include automatic scrolling at the bottom of a page [26], switching focus to the currently viewed window [13] as well as displaying translations when reading [38].

In summary, the potential of gaze to address contextual requirements has already been identified and fellow researchers have, for example proposed to control a (mouse) cursor with gaze to empower disabled users. In our best of knowledge, and surprisingly, the potential of gaze to trigger help in a fashion similar but more adapt touch interface designs has not been explicitly identified and its UX has not yet been explored in a systematic manner. a)

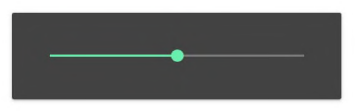

c)

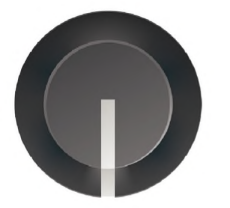

d) b)
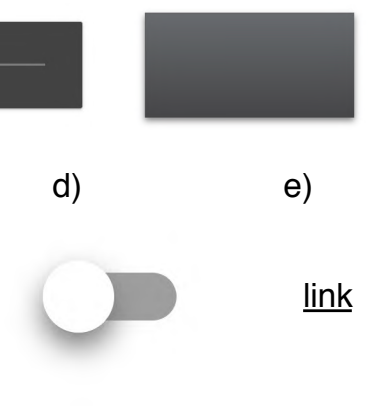

e)

link
Figure 1: Overview of graphical user interface elements a) slider, b) button without label, c) a knob, d) a switch, and e) a weblink/hyperlink, for which affordance communication probes (i.e., animations) were prepared.

\section{USER STUDY}

The main goal of the user study was to explore the potential of a gaze-based technique (i.e., gazeover) to trigger contextual help for users (i.e., signifying affordances) of interface elements on a screen. Since the well-known mouseover technique served as inspiration, we decided to use the UX of mouseover as a control condition.

\subsection{Prototype and Probes}

In order to provide exemplary experiences of gaze-triggered help in communicating "affordances" we set up a prototype system using the off-the-shelf Eye Tribe eye-tracker ${ }^{1}$. The Eye Tribe software, which comes with the hardware provides a feature for calibration. Another feature of the Eye Tribe software allows to set gaze (i.e., gaze position) as input to control the mouse pointer (and an option to hide the graphical mouse pointer), which provides the technical foundation to implement the gazeover experience.

We prepared four animations (based on Javascript and CSS3) as probes and integrated them into websites (with each website showing one interface element), which could be switched through by pressing a key on the keyboard. We chose a small set of typical interface elements (see Figure 1), which implemented a direct manipulation [35] concept and which we believed would be sufficient in testing the technique's effect on UX. Each animation could be triggered through a mouseover Javascript event, and each animation provided "feedforward" information, showing how the element would look when being manipulated (e.g., the slider would slightly move to the left and to the right and then back, the knob would slightly rotate and move back). In contrast to the other interface elements, a gazeover/mouseover event on the text link would cause a standard tooltip behavior (i.e., change the font color and a tip would pop up showing the exact URL of target site).

\footnotetext{
${ }^{1}$ http://theeyetribe.com
} 
a)

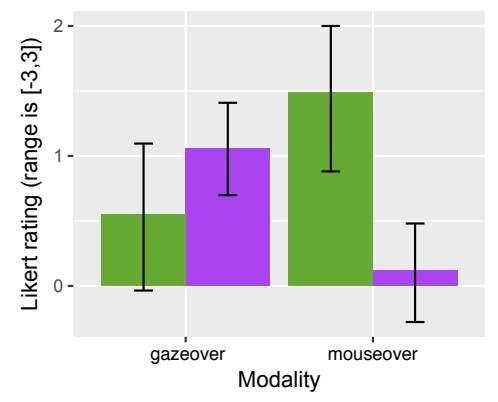

b)

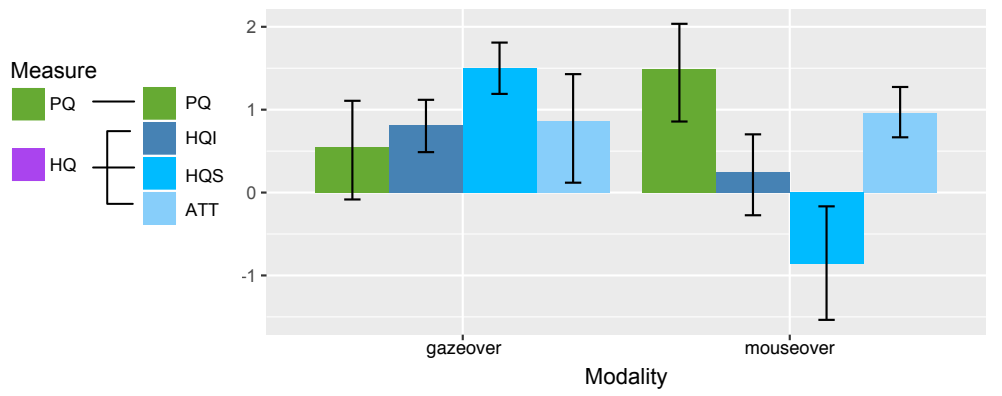

Figure 2: Overview of results, considering a) the two main dimensions pragmatic quality (PQ) and hedonic quality (HQ), and b) showing in detail sub-scales of the hedonic quality.Error bars denote $95 \%$ confidence intervals.

\subsection{Procedure}

Twelve participants (2f) were recruited from a university campus for a within subjects study. All participants were tech savvy owning at least one touch device. Each participant was asked to test both interaction techniques (in counter balanced order). Figure 3 depicts both techniques and the setup. Participants could explore each interface element for as long as they wanted, triggering each animation multiple times until they had a good grasp of the technique and how they felt about it. After each modality participant's were asked to provide ratings based on the attrakDiff questionnaire [17]. attrakDiff measures two dimensions of UX (i) pragmatic quality (i.e., traditional usability) and (ii) hedonic quality (e.g, how attractive and desirable a design is perceived). The HQS measure is associated with a product's perceived capability to satisfy a person's desire of self-improvement, the HQI measure is about a product's perceived capability to communicate others a valuable identity, and ATT measures general attractiveness of a product[17]. At the end of the study, a brief semi-structured interview was conducted with each participant to be able to clarify/identify possible reasons for participants' ratings in a post-hoc qualitative analysis.

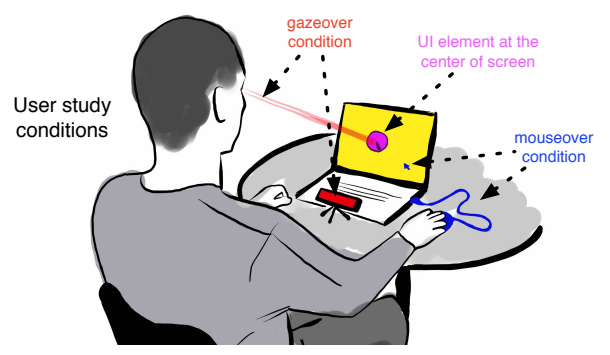

Figure 3: Overview of the setup and modalities.

\subsection{Results}

In the following, we first provide frequency plots of participants' scores, showing possible trends in the data and then results of statistical tests are provided, clarifying the significance of any differences in participants' scores considering the two interaction modalities.
3.3.1 General trends. Figure 2a summarizes participants' scores on pragmatic quality $(\mathrm{PQ})$ and hedonic quality $(\mathrm{HQ})$ associated with both modalities (i.e., gazeover, and mouseover) to trigger the same kind of help based on the aforementioned list of interface element probes. It seems that the gazeover modality received higher scores in HQ and lower scores in PQ than mouseover. Figure $2 \mathrm{~b}$ presents the breakdown of HQ in its three sub-scales HQI, HQS, and ATT. HQI and HQS scores seem higher in average for gazeover, and the difference in ATT scores seems small.

3.3.2 Statistical analysis. In order to validate the statistical significance of differences in participants' scores associated with modality, four (paired) t-tests were conducted. We found a significant main effect of modality on HQ $(t=4.11, p=.002, r=.78)$. The effect of modality on PQ was non-significant $(\mathrm{t}=-2.17, \mathrm{p}=.052, \mathrm{r}=.55)$. Considering the sub-scales of HQ, modality's effect on HQS $(t=5.09$, $\mathrm{p}<.001, \mathrm{r}=.54)$ was significant. Modality's effect on HQI $(\mathrm{t}=2.01$, $\mathrm{p}=.069, \mathrm{r}=.84)$ and ATT $(\mathrm{t}=-.33, \mathrm{p}=.75, \mathrm{r}=.10)$ was non-significant.

In summary, while participants scores on $\mathrm{PQ}$ were higher for mouseover the difference between mouseover and gazeover was not significant. Considering HQ, gazeover received significant higher scores than mouseover. The difference in HQ between the modalities seems mostly related to the difference in HQ's sub-scale HQS, which means that gazeover's perceived capability to satisfy participants' desire of self-improvement was significantly higher. Figure 4 provides in details ratings for all 28 items of the attrakDiff, showing how each item contributes to PQ and sub-scales of HQ.

3.3.3 Analysis of qualitative data. At the end of the study, we asked each participant to provide an overall preference and reasons for their choice. Six participants clearly stated that they would prefer overall the mouseover technique, arguing that gazeover felt exhausting. Participants stated, for example, "You have to be very concentrated all the time", "exhausting, because one is not allowed to move and may otherwise have to calibrate again", another participant mentioned "at its [gaze technologies'] current [development] state, you have to be very mindful". Two participants preferred overall gazeover, but argued that the technology needs to improve and be more robust. The rest of the participants argued that their preference would depend on the specific use context. 


\begin{tabular}{|l|}
\hline technical - human \\
\hline complicated - simple \\
\hline impractical - practical \\
\hline cumbersome - straightforward \\
\hline unpredictable - predictable \\
\hline confusing - clearly structured \\
\hline unruly - manageable \\
\hline isolating -connective \\
\hline unprofessional - professional \\
\hline tacky - stylish \\
\hline cheap - premium \\
\hline alienating integrating \\
\hline separates me - brings me closer \\
\hline unpresentable - presentable \\
\hline conventional- inventive \\
\hline unimaginative - creative \\
\hline cautious - bold \\
\hline conservative - innovative \\
\hline dull - captivating \\
\hline undemanding - challenging \\
\hline ordinary - novel \\
\hline unpleasant - pleasant \\
\hline ugly - attractive \\
\hline disagreeable - likable \\
\hline rejecting - inviting \\
\hline bad - good \\
\hline repelling - appealing \\
\hline discouraging - motivating \\
\hline
\end{tabular}

mouseover

gazeover

Error bars/rectangles denote $95 \%$

Figure 4: Overview of all items of the attrakDiff questionnaire contributing to all four constructs PQ, HQI, HQs, and ATT

Considering gazeover, participants mentioned repeatedly negative performance issues with the technology and how they experienced inconsistence in the pointing performance and that achieving good performance required them to put too much effort. On the other hand, they argued that gazeover felt more natural and fast. One participant stated "one feels closer to the machine".

\section{DISCUSSION}

Today's graphical interfaces are increasingly designed for touchbased interaction, implementing (often) a concept of direct manipulation and "natural" interaction. Benefits of "natural' or realitybased interfaces (e.g., [21]), which, for example visually "replicate/represent" physical and material behaviors of real objects include familiarity with affordances based on previous knowledge. However, using metaphors and mappings for graphcial designs that are inspired by reality and consistent over cultures and individual skills will always be a challenge and user's will benefit from help-triggering techniques. Furthermore, a strive towards designing "natural" interfaces will restrict a designers (aesthetic) expressiveness and potentially limit the creation and (user acceptance) of new interface elements.

While mouse-based interaction is becoming a relict of the past, we have motivated our work by arguing that tooltips associated with mouseover events have been successful in helping users to explore (complex) graphical interfaces' functionality and that similar helpful techniques are missing for many interfaces designed for touch. Many fellow researchers have already identified the potential of eye tracking and gaze as an interaction modality and have been proposing new techniques to improve the interaction with graphical interfaces. While the body of related work is very large, in our best of knowledge the user experience of gaze as an interaction technique has received less attention and, so far, and surprisingly the user experience of mouseover and gazeover have not been explored in a systematic manner. Our intention was to contribute in closing this gap in research.

We have shown (and learned) that gazeover as an interaction technique to trigger "interaction help" is associated with significantly higher hedonic qualities than mouseover. Moreover, the gazeover technique seems to have the potential to satisfy users' desire of self-improvement (i.e., HQS), and thus, may improve feelings associated with competence and flourishing. We believe gazeover could, for example benefit future tourists when integrated in multimodal and multilingual services/interfaces (e.g., [5]).

The work we presented in this paper faces some limitations, which should be considered when interpreting results. First, results are specific to the tracking technology used. Eye tracking technology is still under development and many off-the-shelf devices such as the Eye Tribe device, which was utilized in the user study still require (i) users to go through a calibration process and (ii) are prone to performance issues caused by, for example, users moving their head. We assume that these issues may have contributed (in a negative way) to ratings for gazeover. Second, to overcome performance issues with eye tracking, we have limited (similar to previous research on gaze-based interaction [25, 27]) the user task to exploring one UI element at a time and using enlarged UI elements.

Despite the aforementioned limitations and since eye tracking solutions are becoming cheaper and mass consumer devices, such as Apple's iPhone X are already being equipped with advanced gaze detection systems, we believe our research is timely and that it will motivate and open the way for new research in gaze-based multimodal interaction. Furthermore, techniques, such as gazeover, which may assist users in exploring functionalities of new interfaces in a safe manner have the potential to (re)empower designers and to contribute in transforming the design space of future graphical interfaces, which, today, seem (strongly and exclusively) constrained by reality inspired metaphors and mappings.

\section{CONCLUSION}

We have argued that an assistive technique, similar to the wellknown mouseover technique, would also benefit today's graphical user interfaces, which are designed for touch and direct manipulation. Inspired by technological advancement and mass market devices being equipped with advanced gaze recognition capabilities, we have proposed the gazeover technique and explored its UX by comparing against mouseover. Our results demonstrate that gazeover is already associated with significantly higher scores in hedonic quality than the mouseover technique. In our future work, we plan to study possibilities to integrate the gazeover technique in mobile interaction scenarios and applications. 


\section{REFERENCES}

[1] Antti Aaltonen, Aulikki Hyrskykari, and Kari-Jouko Räihä. 1998. 101 Spots, or How Do Users Read Menus?. In Proceedings of the SIGCHI Conference on Human Factors in Computing Systems (CHI '98). ACM Press/Addison-Wesley Publishing Co., New York, NY, USA, 132-139. https://doi.org/10.1145/274644.274664

[2] Sharifa Alghowinem, Majdah AlShehri, Roland Goecke, and Michael Wagner. 2014. Exploring Eye Activity as an Indication of Emotional States Using an EyeTracking Sensor. Springer International Publishing, Cham, 261-276. https //doi.org/10.1007/978-3-319-04702-7_15

[3] Ilhan Aslan and Elisabeth André. 2017. Pre-touch Proxemics: Moving the Design Space of Touch Targets from Still Graphics Towards Proxemic Behaviors. In Proceedings of the 19th ACM International Conference on Multimodal Interaction (ICMI 2017). ACM, New York, NY, USA, 101-109. https://doi.org/10.1145/3136755. 3136808

[4] Ilhan Aslan, Martin Murer, Verena Fuchsberger, Andrew Fugard, and Manfred Tscheligi. 2013. Drag and Drop the Apple: The Semantic Weight of Words and Images in Touch-based Interaction. In Proceedings of the 7th International Conference on Tangible, Embedded and Embodied Interaction (TEI '13). ACM, New York, NY, USA, 159-166. https://doi.org/10.1145/2460625.2460650

[5] Ilhan Aslan, Feiyu Xu, Hans Uszkoreit, Antonio Krüger, and Jörg Steffen. 2005. COMPASS2008: Multimodal, Multilingual and Crosslingual Interaction for Mobile Tourist Guide Applications. In Intelligent Technologies for Interactive Entertainment, Mark Maybury, Oliviero Stock, and Wolfgang Wahlster (Eds.). Springer Berlin Heidelberg, Berlin, Heidelberg, 3-12.

[6] Andreas Bulling, Jamie A. Ward, Hans Gellersen, and Gerhard Tröster. 2009. Eye Movement Analysis for Activity Recognition. In Ubiquitous Computing (UbiComp), Conference Proceedings. ACM, New York, New York, USA, 41-50. https://doi.org/10.1145/1620545.1620552

[7] Guy Thomas Buswell. 1935. How People Look at Pictures: A Study of the Psychology of Perception in Art. University of Chicago Press, Chicago, Illinois.

[8] S. S. Mozafari Chanijani, S. S. Bukhari, and A. Dengel. 2015. Analysis of text layout quality using wearable eye trackers. In 2015 IEEE International Conference on Multimedia Expo Workshops (ICMEW). 1-6. https://doi.org/10.1109/ICMEW. 2015.7169792

[9] Laura Cowen, Linden J.s Ball, and Judy Delin. 2002. An Eye Movement Analysis of Web Page Usability. In People and Computers XVI - Memorable Yet Invisible, Xristine Faulkner, Janet Finlay, and Françoise Détienne (Eds.). Springer London, London, 317-335. https://doi.org/10.1007/978-1-4471-0105-5_19

[10] Michael Dietz, Maha El Garf, Ionut Damian, and Elisabeth André. 2016. Exploring Eye-Tracking-Driven Sonification for the Visually Impaired. In Proceedings of the 7 th Augmented Human International Conference 2016 (AH '16). ACM, New York, NY, USA, Article 5, 8 pages. https://doi.org/10.1145/2875194.2875208

[11] Michael Dietz, Daniel Schork, and Elisabeth André. 2016. Exploring Eye-TrackingBased Detection of Visual Search for Elderly People. In Intelligent Environments (IE), Conference Proceedings. IEEE, 151-154. https://doi.org/10.1109/IE.2016.32

[12] Michael Dietz, Daniel Schork, Ionut Damian, Anika Steinert, Marten Haesner, and Elisabeth André. 2017. Automatic Detection of Visual Search for the Elderly using Eye and Head Tracking Data. KI - Künstliche Intelligenz 31, 4 (01 Nov 2017), 339-348. https://doi.org/10.1007/s13218-017-0502-z

[13] David Fono and Roel Vertegaal. 2005. EyeWindows: Evaluation of Eye-controlled Zooming Windows for Focus Selection. In Proceedings of the SIGCHI Conference on Human Factors in Computing Systems (CHI '05). ACM, New York, NY, USA 151-160. https://doi.org/10.1145/1054972.1054994

[14] William W. Gaver. 1991. Technology Affordances. In Proceedings of the SIGCHI Conference on Human Factors in Computing Systems (CHI '91). ACM, New York, NY, USA, 79-84. https://doi.org/10.1145/108844.108856

[15] Teresia Gowases, Roman Bednarik, and Markku Tukiainen. 2008. Gaze vs. mouse in games: The effects on user experience. (2008).

[16] John Paulin Hansen, Anders Sewerin Johansen, Dan Witzner Hansen, Kenji Itoh, and Satoru Mashino. 2003. Command without a click: Dwell time typing by mouse and gaze selections. In Proceedings of Human-Computer Interaction-INTERACT. 121-128.

[17] Marc Hassenzahl, Franz Koller, and Michael Burmester. 2008. Der User Experience (UX) auf der Spur: Zum Einsatz von www. attrakdiff. de. Tagungsband UP08 (2008).

[18] Marc Hassenzahl, Axel Platz, Michael Burmester, and Katrin Lehner. 2000. Hedonic and ergonomic quality aspects determine a software's appeal. In $\mathrm{CHI}$.

[19] Edwin L. Hutchins, James D. Hollan, and Donald A. Norman. 1985. Direct Manipulation Interfaces. Hum.-Comput. Interact. 1, 4 (Dec. 1985), 311-338. https: //doi.org/10.1207/s15327051hci0104_2

[20] T. E. Hutchinson, K. P. White, W. N. Martin, K. C. Reichert, and L. A. Frey. 1989 Human-computer interaction using eye-gaze input. IEEE Transactions on Systems, Man, and Cybernetics 19, 6 (Nov 1989), 1527-1534. https://doi.org/10.1109/21. 44068

[21] Robert J.K. Jacob, Audrey Girouard, Leanne M. Hirshfield, Michael S. Horn, Orit Shaer, Erin Treacy Solovey, and Jamie Zigelbaum. 2008. Reality-based Interaction: A Framework for post-WIMP Interfaces. In Proceedings of the SIGCHI Conference on Human Factors in Computing Systems (CHI '08). ACM, New York, NY, USA, 201-210. https://doi.org/10.1145/1357054.1357089

[22] Robert J. K. Jacob. 1990. What You Look at is What You Get: Eye Movementbased Interaction Techniques. In Proceedings of the SIGCHI Conference on Human Factors in Computing Systems (CHI '90). ACM, New York, NY, USA, 11-18. https: //doi.org/10.1145/97243.97246

[23] Marcel Adam Just and Patricia A. Carpenter. 1976. Eye fixations and cognitive processes. Cognitive Psychology 8, 4 (1976), 441-480. https://doi.org/10.1016/ 0010-0285(76)90015-3

[24] Chris L Kleinke. 1986. Gaze and Eye Contact: A Research Review. Psychological bulletin 100, 1 (1986), 78. https://doi.org/10.1037/0033-2909.100.1.78

[25] Manu Kumar, Andreas Paepcke, and Terry Winograd. 2007. EyePoint: Practical Pointing and Selection Using Gaze and Keyboard. In Proceedings of the SIGCHI Conference on Human Factors in Computing Systems (CHI '07). ACM, New York, NY, USA, 421-430. https://doi.org/10.1145/1240624.1240692

[26] Manu Kumar and Terry Winograd. 2007. Gaze-enhanced Scrolling Techniques. In Proceedings of the 20th Annual ACM Symposium on User Interface Software and Technology (UIST '07). ACM, New York, NY, USA, 213-216. https://doi.org/10. 1145/1294211.1294249

[27] Chris Lankford. 2000. Effective Eye-gaze Input into Windows. In Proceedings of the 2000 Symposium on Eye Tracking Research \& Applications (ETRA '00). ACM, New York, NY, USA, 23-27. https://doi.org/10.1145/355017.355021

[28] Päivi "Majaranta, Ulla-Kaija Ahola, and Oleg Špakov. 2009. Fast Gaze Typing with an Adjustable Dwell Time. In Proceedings of the SIGCHI Conference on Human Factors in Computing Systems (CHI '09). ACM, New York, NY, USA, 357-360. https://doi.org/10.1145/1518701.1518758

[29] Päivi Majaranta and Andreas Bulling. 2014. Eye Tracking and Eye-Based HumanComputer Interaction. Springer London, London, 39-65. https://doi.org/10.1007/ 978-1-4471-6392-3 3

[30] Julio C. Mateo, Javier San Agustin, and John Paulin Hansen. 2008. Gaze Beats Mouse: Hands-free Selection by Combining Gaze and Emg. In CHI '08 Extended Abstracts on Human Factors in Computing Systems (CHI EA '08). ACM, New York, NY, USA, 3039-3044. https://doi.org/10.1145/1358628.1358804

[31] Bernhard Maurer, Ilhan Aslan, Martin Wuchse, Katja Neureiter, and Manfred Tscheligi. 2015. Gaze-Based Onlooker Integration: Exploring the In-Between of Active Player and Passive Spectator in Co-Located Gaming. In Proceedings of the 2015 Annual Symposium on Computer-Human Interaction in Play (CHI PLAY '15). ACM, New York, NY, USA, 163-173. https://doi.org/10.1145/2793107.2793126

[32] Darius Miniotas, Oleg Špakov, Ivan Tugoy, and I. Scott MacKenzie. 2006. Speechaugmented Eye Gaze Interaction with Small Closely Spaced Targets. In Proceedings of the 2006 Symposium on Eye Tracking Research \&Amp; Applications (ETRA '06). ACM, New York, NY, USA, 67-72. https://doi.org/10.1145/1117309.1117345

[33] Aanand Nayyar, Utkarsh Dwivedi, Karan Ahuja, Nitendra Rajput, Seema Nagar, and Kuntal Dey. 2017. OptiDwell: Intelligent Adjustment of Dwell Click Time. In Proceedings of the 22Nd International Conference on Intelligent User Interfaces (IUI '17). ACM, New York, NY, USA, 193-204. https://doi.org/10.1145/3025171.3025202

[34] Don Norman. 2013. The design of everyday things: Revised and expanded edition. Basic Books (AZ).

[35] Ben Shneiderman. 1981. Direct Manipulation: A Step Beyond Programming Languages (Abstract Only). SIGSOC Bull. 13, 2-3, 143-. https://doi.org/10.1145/ 1015579.810991

[36] J. David Smith and T. C. Nicholas Graham. 2006. Use of Eye Movements for Video Game Control. In Proceedings of the 2006 ACM SIGCHI International Conference on Advances in Computer Entertainment Technology (ACE '06). ACM, New York, NY, USA, Article 20. https://doi.org/10.1145/1178823.1178847

[37] Sophie Stellmach and Raimund Dachselt. 2012. Look \&\#38; Touch: Gazesupported Target Acquisition. In Proceedings of the SIGCHI Conference on Human Factors in Computing Systems (CHI '12). ACM, New York, NY, USA, 2981-2990. https://doi.org/10.1145/2207676.2208709

[38] Takumi Toyama, Daniel Sonntag, Andreas Dengel, Takahiro Matsuda, Masakazu Iwamura, and Koichi Kise. 2014. A Mixed Reality Head-mounted Text Translation System Using Eye Gaze Input. In Proceedings of the 19th International Conference on Intelligent User Interfaces (IUI '14). ACM, New York, NY, USA, 329-334. https: //doi.org/10.1145/2557500.2557528

[39] Colin Ware and Harutune H. Mikaelian. 1987. An Evaluation of an Eye Tracker As a Device for Computer Input2. In Proceedings of the SIGCHI/GI Conference on Human Factors in Computing Systems and Graphics Interface (CHI '87). ACM, New York, NY, USA, 183-188. https://doi.org/10.1145/29933.275627

[40] Yanxia Zhang, Sophie Stellmach, Abigail Sellen, and Andrew Blake. 2015. The Costs and Benefits of Combining Gaze and Hand Gestures for Remote Interaction. In 15th IFIP TC 13 International Conference on Human-Computer InteractionINTERACT 2015 - Volume 9298. Springer-Verlag New York, Inc., New York, NY, USA, 570-577. https://doi.org/10.1007/978-3-319-22698-9 39

[41] W. L. Zheng, B. N. Dong, and B. L. Lu. 2014. Multimodal emotion recognition using EEG and eye tracking data. In 2014 36th Annual International Conference of the IEEE Engineering in Medicine and Biology Society. 5040-5043. https://doi.org/ 10.1109/EMBC.2014.6944757 